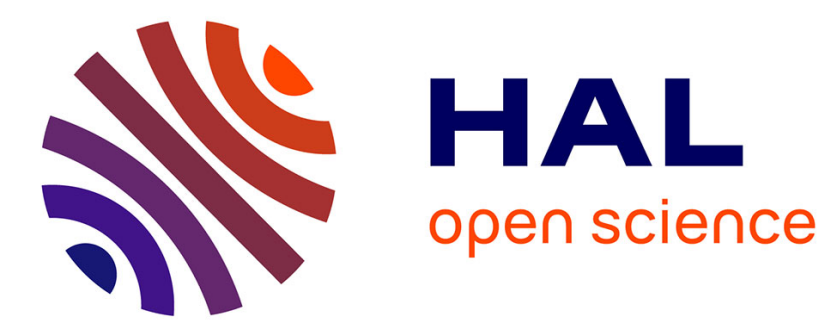

\title{
Studying Navigation as a Form of Interaction: a Design Approach for Social Robot Navigation Methods
}

\author{
Philip Scales, Olivier Aycard, Véronique Auberge
}

\section{To cite this version:}

Philip Scales, Olivier Aycard, Véronique Auberge. Studying Navigation as a Form of Interaction: a Design Approach for Social Robot Navigation Methods. International Conference on Robotics and Automation (ICRA 2020), 2020, Paris, France. hal-02541820

\section{HAL Id: hal-02541820 \\ https://hal.science/hal-02541820}

Submitted on 21 Apr 2020

HAL is a multi-disciplinary open access archive for the deposit and dissemination of scientific research documents, whether they are published or not. The documents may come from teaching and research institutions in France or abroad, or from public or private research centers.
L'archive ouverte pluridisciplinaire HAL, est destinée au dépôt et à la diffusion de documents scientifiques de niveau recherche, publiés ou non, émanant des établissements d'enseignement et de recherche français ou étrangers, des laboratoires publics ou privés. 


\title{
Studying Navigation as a Form of Interaction: a Design Approach for Social Robot Navigation Methods*
}

\author{
Philip Scales $^{1}$, Olivier Aycard ${ }^{2}$, Véronique Aubergé ${ }^{3}$
}

\begin{abstract}
Social Navigation methods attempt to integrate knowledge from Human Sciences fields such as the notion of Proxemics into mobile robot navigation. They are often evaluated in simulations, or lab conditions with informed participants, and studies of the impact of the robot behavior on humans are rare. Humans communicate and interact through many vectors, among which are motion and positioning, which can be related to social hierarchy and the socio-physical context. If a robot is to be deployed among humans, the methods it uses should be designed with this in mind. This work acts as the first step in an ongoing project in which we explore how to design navigation methods for mobile robots destined to be deployed among humans. We aim to consider navigation as more than just a functionality of the robot, and to study the impact of robot motion on humans. In this paper, we focus on the personfollowing task. We selected a state of the art person-following method as the basis for our method, which we modified and extended in order for it to be more general and adaptable. We conducted pilot experiments using this method on a real mobile robot in ecological contexts. We used results from the experiments to study the Human-Robot Interaction as a whole by analysing both the person-following method and the human behavior. Our preliminary results show that the way in which the robot followed a person had an impact on the interaction that emerged between them.
\end{abstract}

\section{INTRODUCTION}

Many companies (such as Amazon, Bossanova, Hease) and researchers in the robotics and IoT fields are increasingly interested in deploying mobile robots in spaces that they will share with humans. Among the few current examples of such robots, there are accounts of people interpreting robot behavior as a cue of interaction and communication processes. Observations in real uses (RELAY room service robot, bomb disposal robot [1], Diya One air purifying robot) show that even if the robot is devoted to service tasks (as opposed to social interaction) it may be perceived as intentionally interacting. This could be seen as an empathic illusion whereby the robot's signals, such as the manner in which it navigates, are interpreted as social signals. Research has also been conducted regarding empathy towards robots [2].

Our review of works observing human behavior when interacting with robots already suggests that the dimensions

\footnotetext{
${ }^{1}$ Philip Scales (philip.scales@univ-grenoble-alpes.fr), Olivier Aycard ${ }^{2}$ (olivier.aycardeimag.fr), and Véronique Aubergé $^{3}$ (veronique. auberge@univ-grenoble-alpes.fr) are with Univ. Grenoble Alpes, CNRS, Grenoble INP, LIG, 38000 Grenoble, France

*This work has been partially supported by MIAI @ Grenoble Alpes, (ANR-19-P3IA-0003)
}

linked to navigation (such as distance, orientation or kinematics) cannot be modeled with pre-determined and fixed constants, even when their values originate from ecological experiments.

It can be supposed that human's social perception of robots will most likely differ from their social perception of other humans, and we claim that understanding how humans will augment their social density with robots (compared to other humans or pets) will require people to have a real cultural experience of large scale robot integration. But it can already be shown that the fact that such navigation dimensions may need to be variable is coherent with our review of a small selection of works on the widely studied domain of proxemics, which relates to dimensions such as movement, touch, distances and gaze between humans. These works show that beyond the purely "technical" reasons for movement and navigation, there is also an aspect of communication and interaction encoded in the variations of these dimensions, even when they are subtle. Furthermore, these works show that the communicative value attributed to such variations depends heavily on culture, situation and the relative roles of the interacting humans. In contrast, current Social Navigation ( $\mathrm{SN}$ ) methods treat such dimensions as constraints, user preferences, or fixed parameters, rarely considering their impact on Human-Robot Interaction (HRI) and the acceptance and evaluation of the robot.

This paper marks the start of an ongoing project to design social navigation methods in an iterative manner, with an emphasis on adaptivity of the method, evolvability of the system, ecological experimentation, and thorough analysis of the experimental data and observed HRI both from the human and robot's perspectives. The methodology used in this paper and that will be used in our future works can be summarized as follows:

1) Design an adaptive method;

2) Confront it to reality through ecological experiments;

3) Analyse the Human-Robot Interaction;

4) Question our hypotheses;

5) Modify system behavior, and start again from 2).

This process during which the navigation architecture is changed according to feedback from experiment and analysis cycles is represented in Fig.1. This methodology goes handin-hand with an adaptable algorithm, and evolvable software 


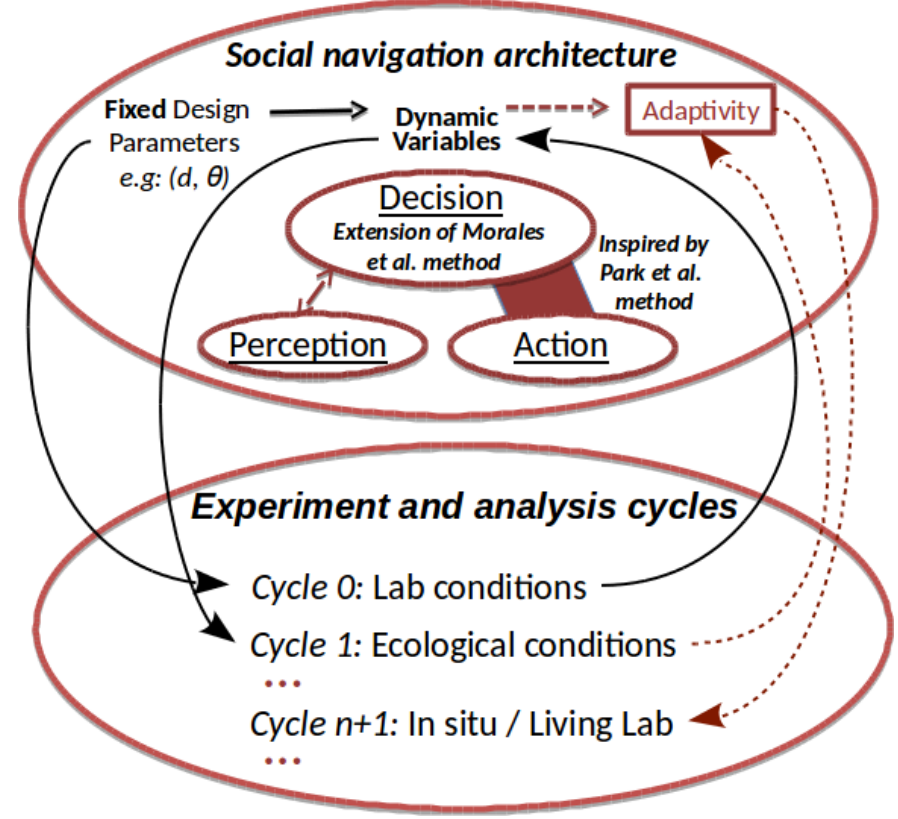

Fig. 1. Our iterative methodology. Solid arrows: cycles 0 and 1 of the methodology, accomplished during this work. Dotted arrows: direction for future works.

and hardware ${ }^{1}$, allowing the robot platform to evolve according to continuous feedback from all concerned parties.

In this paper, we propose to first experimentally verify whether the dimensions controlled by navigation algorithms should be adaptable to the situation as well as to the shift in the social perception of the robot as it is progressively integrated into human's social space (cycles 0 and 1 in Fig.1). Among the various facets of mobile robot navigation, in this paper, we choose to study this question through the example of the person-following task, where a robot must maintain the position specified by any point $(d, \theta)$ w.r.t the person. Our contributions are as follows:

- We describe our adaptable person-following architecture, combining and extending notions from previous works [3] and [4].

- We discuss the impact on HRI of the position of a robot w.r.t the followed person based on results of preliminary experiments performed on a real mobile robot in ecological conditions.

\section{RELATED WORKS}

Human perception of physical and social variables is known to be influenced by environmental and situational factors [5], [6], [7], [8], [9]. HRI studies on gestures [10], speech interaction [11], gaze and position [12] have shown that the way people interact with and perceive a robot can depend on the type of experimental conditions (lab or ecological), as well as how the robot behaves, and the situation or context in which it is deployed [13]. Experimentation in

\footnotetext{
${ }^{1}$ The mobile robot platform we use is designed, built, and maintained locally, and can accommodate various sensor and actuator configurations (https://github.com/fabMSTICLig/RobAIR).
}

ecological conditions followed by a critical analysis of the human-robot system is therefore key to truly test the robot and understand how people react to it, what people think of it, and how it should evolve. Positioning and movement are a known interaction and communication vector in HumanHuman relationships [6]. Various other vectors have received attention from the HRI field as seen in works studying gaze [14], intention [15], engagement [16], robot initiative [17], reaction time to touch stimuli [18]. In comparison, few works study the effect of varying the dimensions controlled by navigation algorithms on a robot's interaction with surrounding humans.

Typical Social Navigation approaches [19], [20], [21], [22], [23] and [24] often improve on traditional humanagnostic navigation by directly integrating an established model from a Human Sciences field, such as the Social Forces Model. Using models taken directly from Human Sciences can lead to promising results, however we shouldn't assume that observations of Human behavior can or should be directly applied to a HRI context [25]. Machine learning methods usually aim to replicate human behavior with a robot and rely on demonstrations [26], [27] or large datasets [28], [29], [30]. If a robotic system is meant to take into account contextual, cultural, social and human factors, then it seems unreasonable to expect to be able to provide training data or demonstrations in sufficient quantities and quality to learn the vast variety of complex, fine-grained behaviors.

Person-following methods generally account for a very limited subset of variables, mostly of a practical, physical nature, as is discussed in a recent literature review on the topic [31]. Typically, algorithms either use the person's current position as the robot's goal [32], [33], or perform a short-term anticipation [34], [35], [36], [4], [3]. In terms of the functionality provided, traditionally the robot is simply behind the person, but other works position the robot at the person's side. This is usually a fixed parameter or a user preference, given that often the algorithmic cost is not affected by the position of the robot. However, the consequences on humans of such differences in how the robot positions itself while following have not been studied.

Few SN works (including person-following) actually deploy and evaluate their method on a real robot in ecological experimental conditions, instead using simulations, experiments in lab-conditions, or using informed participants. Even fewer attempt to understand the impact of the robot on the humans. Furthermore, the dimensions controlled by navigation algorithms are rarely considered as a vector for interaction or communication. For these reasons, we use our methodology to develop a general, flexible SN architecture, and perform ecological experiments to study and evaluate it.

\section{PERSON-FOLlOWING ARCHITECTURE}

\section{A. Overview}

In many works using utility functions including the work by Park et al.[4], the utility function is used solely to define the robot's behavior, and does not attempt to model the person's behavior. The Joint Utility formulation of Morales 
et al.[3] allows to model the fact that the person's behavior can depend on the robot's behavior and vice-versa. We base our person-following method (see Fig.2) on the work of Morales et al. [3] which was the closest to our vision. It is a form of sampling-based local planner where a utility function is used to score states sampled from the joint state space of the robot and person. Among a set of reachable states, the planner selects the state that maximises the utility function which takes into account dimensions related both to the person and the robot such as their velocities, accelerations, the robot-person distance $d_{r p}$, and the relative angle $\theta_{r p}$.

In this paragraph, we summarize the Morales et al. approach and refer readers to [3] for details. We use the term Agent to refer to a physical entity. We consider two Agents: the robot $R$ and the followed person $P$. First, raw data from the robot's sensors are processed by perception modules. The perception modules output information regarding the environment and the current state of each Agent, denoted $X_{R}^{t}$ and $X_{P}^{t}$. Then, the decision module uses this information to determine which action the robot should perform in order to reach the best Future State. The right branch of the decision module (Fig.2) can be seen as specifying what each Agent aims to do given their current Agent States, in the form of their respective Utility Functions $a u_{R}$ and $a u_{P}$. The left branch involves generating a representation of what the robot and the person could physically do in the form of Joint Future States, each of which is a tuple consisting of a robot and a person Future State. The results of these two branches are combined in the Joint Utility Evaluation step to select what the robot should do, and what the person is predicted to do in the form of the Joint Future State which maximises the sum of their utility functions $a u_{R}$ and $a u_{P} . a u_{R}$ and $a u_{P}$ are themselves composed of multiple individual Utilities, each of which maps the value of a single dimension (for example $d_{r p}$ or $\theta_{r p}$ ) to a score. Given the optimal Joint Future State, an Action module computes the control variable values to be passed to the motors to enable the robot to reach that state. In the following subsections, we detail the two modules of our architecture which differ from [3] and motivate our choices.

\section{B. Agent Future States Generation}

1) Method: The inputs to this step are the current Agent States $X_{R}^{t}$ and $X_{P}^{t}$. We use Future State to refer to a possible Agent State at a given time in the future $t+\delta t$. Given its current state, a Future State for an Agent is a new Agent State resulting from performing a feasible action over a finite time horizon $\delta t$. An action is a set of values of control variables (linear and angular velocity, $(v, \omega)$ ) to be applied by an Agent during the time horizon. We discretely sample the ranges of $v$ and $\omega$, and construct the set of all feasible $(v, \omega)$ pairs. A $(v, \omega)$ pair is feasible if it complies with the Agent's kinematics constraints, and it does not lead to a collision. To generate the set of Future States of an Agent, we solve the forward kinematics for each action, described by a $(v, \omega)$ pair. The robot Agent uses

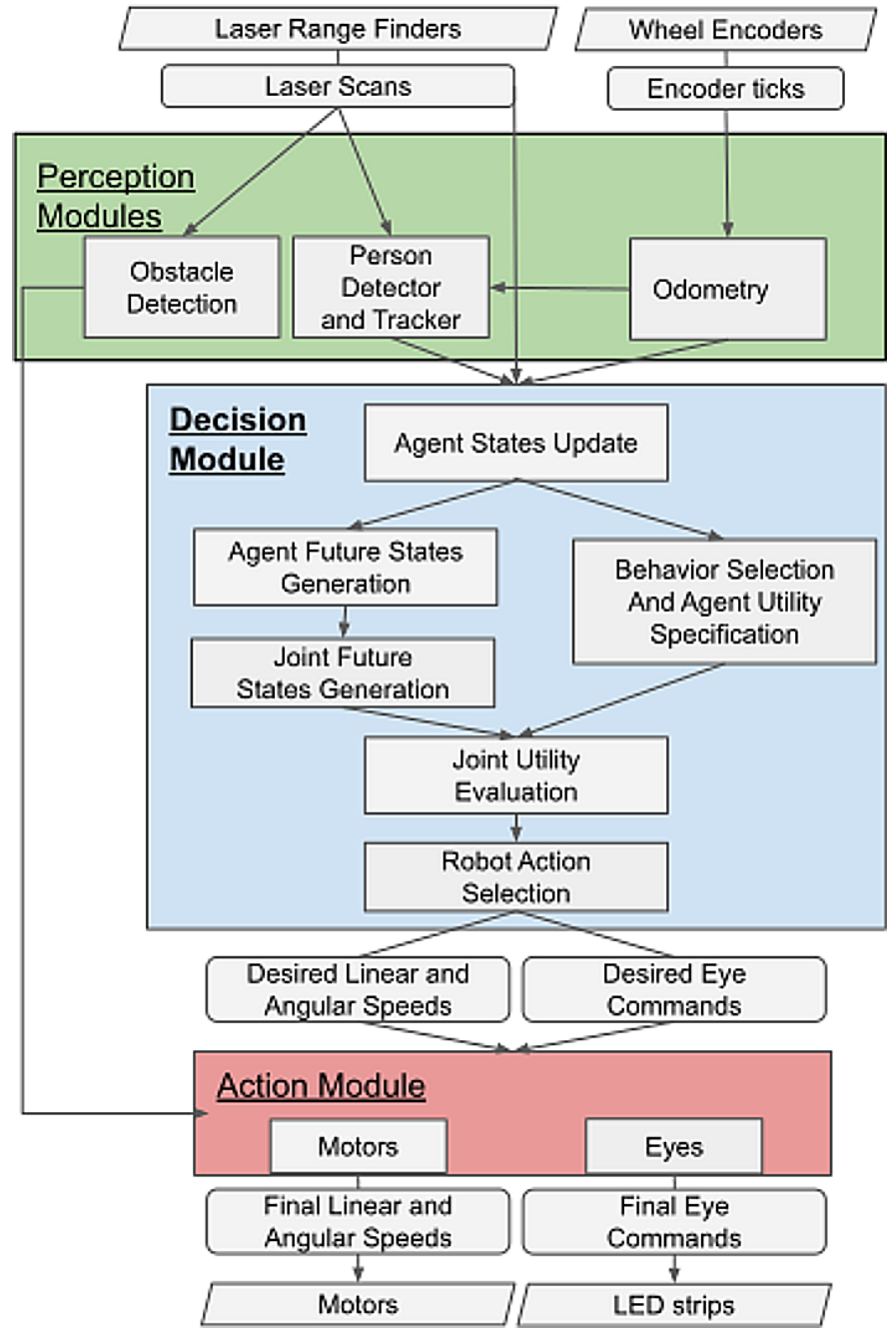

Fig. 2. Our person-following architecture and control flow.

the same kinematics model as the one which is intended to be used to control the physical robot. The outputs of this step are each Agent's finite set of Future States, in our case, $F S_{R}=\left\{X_{R, 1}^{t+\delta t}, X_{R, 2}^{t+\delta t}, \ldots X_{R, n}^{t+\delta t},\right\}$ and similarly, $F S_{P}$.

2) Motivation: In [3], few details are given about the robot's controller, and the model of robot movement used in their decision module is not the same as the model used in their action module, given that once the state is selected, they use a separate trajectory-following algorithm in order to perform low-level control ${ }^{2}$. This leads to a discrepancy between the action used to generate the Future State evaluated by the utility function, and the action that the real robot will perform. Our aim is to be able to precisely specify robot behavior according to the utility function. In order to achieve this, we take an approach more similar to the integrated planning and control of [4] in that our Future States are generated using the same model which the lowlevel robot controller will use to reach them.

\footnotetext{
${ }^{2}$ This information was not detailed in the article, and was obtained by contacting the main author of [3].
} 


\section{Behavior Selection and Agent Utility Specification}

1) Method: The inputs to this step are the current Agent States $X_{R}^{t}$ and $X_{P}^{t}$. We use Agent Utility to refer to a utility function that associates a score to a Joint Future State. The robot's Agent Utility $a u_{R}$ describes how the robot should act. The person's Agent Utility $a u_{P}$ predicts how the person may act. This step serves to dynamically specify the person and robot's Agent Utilities according to $X_{R}^{t}$ and $X_{P}^{t}$, as opposed to it being fixed when designing the method.

We use Behavior to refer to one of several possible ways in which the robot or person can act. The Behavior of the robot and person can be different from each other and can change over time. The robot and the person Agents each use a finite state machine where a state represents one of their Behaviors, and state transitions are defined with respect to any of the Agent's Agent States. The robot and person Behaviors, $B_{R}$ and $B_{P}$, specify the terms and parameters of their respective Agent Utilities. [37] proposed a similar concept for a specific case of person-following, however we extend this notion by also specifying how the terms and parameters of each Behavior should change over time, or as functions of $X_{R}^{t}$ and $X_{P}^{t}$. The outputs of this step are the Agent Utilities for the person and the robot.

2) Motivation: We use Utility in a similar way to [3] to refer to a single-variable utility function assigned to an Agent that associates a score to a quantity that depends on a Joint Future State. A Utility $U$ may be defined on a quantity contained in an Agent State, such as linear speed of the robot $\left(U_{R}^{V}\right)$, or on a value resulting from a computation such as the relative distance between the robot and the person $\left(U_{R}^{d_{R P}}\right)$. A Utility essentially encodes the preferred, desired, or ideal values of the quantity it measures. For instance, if we model $U_{R}^{d_{R P}}$ as a bell curve centered on the value $1.0 \mathrm{~m}$ and use this Utility as the robot's Agent Utility, then the robot will select actions that drive it towards a position one meter away from the person. If we change the center value, we change this behavior. Similarly, when an Agent Utility is composed of more than one Utility, the relative weights of each Utility have an impact on how the robot behaves.

In [3], each Utility of an Agent Utility is fixed. The parameters and weights are not continuously changed at run-time according to changes in the Agent States or the environment. Our goal is to obtain a method which is evolvable, meaning we can implement new functionalities without starting from scratch. We also aim for the method to be adaptable so that the robot's behavior can be adjusted in real-time depending on which task the robot is performing, the context, and the humans which are present. As a step towards achieving this, we extend the usage of Utilities of [3] by proposing to specify Utility parameters and weights dynamically according to changes in Agent States. If the robot's Utility parameters can be specified dynamically during operation, rather than as part of the design phase, the robot can better adjust its person-following behavior to the situation.

In order to change a parameter of a Utility, we suggest to define a relationship between the parameter and a quantity related to an Agent State. For example, we may want the robot to move in the same direction as the person when they are moving side by side, as well as staying close by. Simply adding a Utility which encourages the robot's heading angle to be the same as the person's leads to an issue. If the robot is initially far from the person, then it makes no sense for the robot to give a large importance to maintaining a similar heading until it is close to the person. With our proposed extension, the weight of the heading angle Utility could be determined according to the robot-person distance to avoid this problem, and smoothly transition from moving towards the person to moving with the person. This parameter modification takes place as part of the Agent Utility Specification step.

\section{IMPLEMENTATION}

We used a RobAIR mobile robot for our implementation and experiments. RobAIR is produced by the FabMSTIC, and serves as a mobile robotics platform for teaching and research. RobAIR is a differential drive wheeled robot, and stands at $1.20 \mathrm{~m}$ high, with a diameter of $0.50 \mathrm{~m}$ at its largest point, at its base. RobAIR is equipped with two forwardfacing Hokuyo URG-04LX-UG01 2D Laser Range Finders (LRF) with a range of $5 \mathrm{~m}$, covering $240^{\circ}$ and updating at $10 \mathrm{~Hz}$. The bottom laser is mounted on the front of the robot $0.10 \mathrm{~m}$ above the ground and $0.35 \mathrm{~m}$ from the center of rotation. The top laser is mounted on top of the robot's head, at a height of $1.20 \mathrm{~m}$ at the center of rotation of the mobile base. A fisheye-lens camera is mounted in RobAIR's "forehead", facing forwards. The on-board processor for RobAIR is a tablet pc, with an i5-6200U processor, which is mounted on RobAIR's "chest". RobAIR is also equipped with LED strips around its head, which are usually used to display eyes. RobAIR's motors allow it to translate at a maximum of $1.1 \mathrm{~m} / \mathrm{s}$, and to rotate at a maximum of $4.5 \mathrm{rad} / \mathrm{s}$.

For the purposes of our preliminary experiments, we used the utility function weights and parameters that were used in [3], excluding the subgoal utility. Parameters for the desired robot-person distance and relative angle were changed during the experiments to obtain different robot following positions. Since RobAIR is a differential drive robot, we implement both Agent Future State Generation (with $\delta t=1.0 \mathrm{~s}$ ) and the low-level controller using the unicycle model. For the person Agent, we approximate their kinematics by using the same model. Perception and Decision modules update at $10 \mathrm{~Hz}$, and the Action module updates at $20 \mathrm{~Hz}$, performing a moving average of the commands given by the Decision module. The architecture was implemented using ROS.

\section{EXPERIMENTS}

Firstly, we present our experiment conducted in lab conditions, similar to those in [3]. Secondly, we present the results of two experiments conducted in ecological conditions ${ }^{3}$ as in the Living Lab method [10]. To this end, we used our dynamic specification of utility parameters

\footnotetext{
${ }^{3}$ Videos of the HRI experiments available at http://ligmembres.imag.fr/aycard/html/Projects/Robair/Scales/scales.html
} 
to switch between the following settings for $\left(d_{r p}, \theta_{r p}\right)$ : setting $0(0.8 \mathrm{~m}, \pi / 2 \mathrm{rad})$ (beside person), and setting 1 $(0.8 \mathrm{~m}, \pi \mathrm{rad})$ (behind person). In both experimental conditions, the robot's maximal speed was limited to $0.6 \mathrm{~m} / \mathrm{s}$.

\section{A. Lab conditions: corridor environment}

The goal of this experiment was both to test the implementation, and to replicate experimental conditions similar to those of [3] as a baseline for how the participants and robot interacted. Our hypothesis was that the position of the robot would not impact how people behaved. Four recruited participants were asked to walk down a corridor and back with the robot. Participants were told whether the robot would position itself beside them (setting $0, n=2$ ) or behind them (setting 1, $n=2$ ). The L-shaped corridor used for this experiment was $20 \mathrm{~m}$ long and $3 \mathrm{~m}$ wide with a $90^{\circ}$ turn at the end. No other people were present. All participants started by walking faster than the robot, and then slowed down to the robot's maximal speed, maintaining a slight lead. The relative distance between the robot and person averaged $1.0 \mathrm{~m}$ as opposed to the desired $0.8 \mathrm{~m}$. From an interaction perspective, we did not observe any difference between when the robot was attempting to be beside or behind the participant.

\section{B. Ecological conditions}

1) Method: The main goal of these two experiments was to study what effect the robot navigation had on people, and whether this changed according to how the robot positioned itself relative to the person when following them. The first was conducted during a public event held in a large crowded gymnasium (see Fig.3), and the second in a mostly empty entrance hall of a research building. Pretext tasks were used to avoid artificially drawing participant's attention to RobAIR's navigation and positioning cues. Each participant performed the task only once, with either setting 0 or 1 . In the first, 6 participants (setting $0: n=3$, setting $1: n=3$ ) had to deliver items carried on the robot to a location, and in the second, 4 participants (setting $0: n=2$, setting 1 : $n=2$ ) had to "show" the robot various points of interest. Participants were random bystanders, and were simply told the pretext task, and that the robot would follow them. They were not told how to walk, nor where the robot would position itself.

2) Results: Using setting 0 (beside), the robot would turn to the right in order to position itself on the right hand side of the person relative to their direction of movement. Very often, participants started moving towards the same direction, somewhat mirroring the robot's change in direction of travel. This resulted in the robot moving even further to the right, given that now it was predicting that the person would keep moving to the right. This often resulted in the person-robot pair going in a completely different direction to what was required to reach the destination. This was observed with all participants using setting 0. Participants were always surprised when told that the robot was trying to position itself at their side at the end of the experiment. Some participants

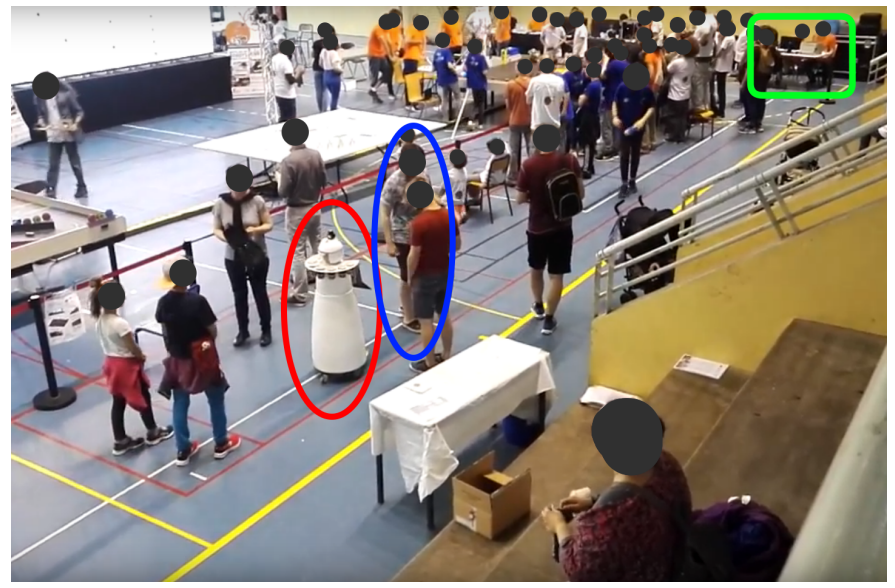

Fig. 3. RobAIR (red) following a person (blue) who was instructed to take RobAIR to a destination (green).

$(n=2)$ tested the robot again after having been told this, and managed to replicate the side-by-side configuration as long as they walked at the pace of the robot. In contrast, using setting 1 (behind), this kind of behavior was not observed. Participants kept walking towards the destination, stopping when they got far from the robot, and waiting for it to catch up before moving again. Some participants walked at a similar pace to that of the robot.

3) Analysis: Two issues arose when using setting 0 (robot beside person). Firstly, people often walked too fast, so the robot could not catch up and position itself at their side. Secondly, all participants using setting $0(n=5)$ seemed to interpret the robot's turning as a sign that the robot was not following them anymore. This illustrates a lack of understanding or misinterpretation of what the robot was trying to achieve, even though participants were told that the robot was going to follow them. This did not occur in our lab-condition experiment, nor in experiments reported in [3] and subsequent works [38], [39] where participants were informed of how they should walk, and how the robot would behave. This confusion did not occur when using setting 1 (robot behind person, $n=5$ ). The ceiling effect of the robot's slow speed compared to human walking speed was interpreted differently depending on the relative distance and angle and seemed tightly linked to the robot's orientation. The difference in participants understanding and behavior resulting from different navigation settings supports our hypothesis, although further experimentation and analysis is required to confirm the effect.

\section{DISCUSSION}

\section{A. Experimental Results}

When performing experiments with participants who were informed about how the robot would follow them, and who were asked to walk slowly, the robot was able to perform close to ideally in terms of its positioning relative to the person both when aiming to follow behind them or at their side. When the same algorithm was used in experiments with uninformed participants, the side-by-side 
following setting resulted in confusion. In both experiments, people were aware that the robot should follow them, and people did not actively try to mislead or run away from the robot. People were still engaged in the person-following activity, but their behavior was different. This illustrates two problems. The first problem is that using constant parameters for the person's utility function does not allow us to capture the difference in their behavior. The second problem is that even if the robot is capable of performing a task (such as following side-by-side), it is useless unless people understand what the robot is trying to accomplish. Otherwise, as was observed, people will make their own conclusions based on the robot's behavior, and assume the robot is not following them.

\section{B. Navigation Algorithm Limitations}

1) Utility Function Formulation: In all experiments, when people suddenly went too far from the robot, the robot stopped making progress towards the person. This was not reported in [3], and we believe this is due to the utility function rewarding states where values of the dimensions are close to the desired value rather than rewarding states which lead to progress towards the desired value. When people walked too far, none of the robot's reachable future states were close enough to the ideal position, so the distance utility score was negligible compared to other utility scores.

2) Tuning Utility weights and Utility function profiles: Determining a utility function as a combination of an arbitrary number of Utilities seems feasible in practice, however the major limitation of such an approach is the choice of parameters and Utility weights. Further problems arise when we consider the possibility of multiple factors influencing the same Utility, for example, the type of environment and the current robot task. Further still, when adding many Utilities and combining them, it may become difficult to determine what effect a given change in the parameters will have on the final outcome.

3) Joint Utility Evaluation: The Joint Utility Evaluation proposed in [3] entails assumptions about Agents cooperating which can be counter-intuitive when combined with the perAgent Utility specification. For example, participants rarely stayed close to the robot, whereas the Utility Function used to predict the person's movements assumed that they would aim to comply with the desired relative distance and angle to the robot.

4) Future State Generation and Control: The decision module often acts in a short-sighted way. Furthermore our method of using the Future State directly as the setpoint for the controller leads to quite unstable motion. It is likely that better results could be obtained by using something more similar to the Model Predictive Controller in [4], with multiple timesteps distributed over a longer time horizon.

\section{CONCLUSION AND FUTURE WORK}

\section{A. Conclusion}

In this work, we adopt a different attitude towards studying mobile robot navigation around humans, inspired by knowl- edge from Human Sciences as well as robotics fields and existing use-cases. We presented our methodology for designing social navigation methods, based on iteration, ecological experiments and thorough analysis of HRI. We extended and generalized existing person-following works in order to suit our need for an adaptable, evolvable architecture. We used our architecture to perform experiments which studied how changing the relative position of a person-following robot impacts the interaction with participants in lab and ecological experimental conditions. Our analysis of the resulting robot behavior also provides some useful insight into some of the limitations of our method. Our results show that the way in which the robot performed its task of person-following lead to different participant behavior in ecological conditions, whereas there was no difference when in lab conditions. Most notably, our preliminary experiments underline a key problem when people interacting with the robot are not told what exactly the robot is trying to achieve. When the robot attempted to follow at their side, we systematically found that people did not understand the robot's goal.

\section{B. Future work}

1) Taking into account Human-Robot comprehension: In our experiments, people rarely understood that the robot was trying to position itself on their right-hand side. As a result, people moved further right, most likely assuming the robot was veering off course or had lost them. The robot adapts itself to the person's new direction of travel, and we enter a "vicious cycle" which stops the pair from reaching their goal. If the robot could realise that the person did not understand its intent, it could adopt a different behaviour to make its intent clear. One possibility is to design a method that takes into account the person's understanding of the robot behavior and intent, as a form of inter-proprioception.

2) Social Cost: A long-term goal of our work is to formulate a form of Social Cost, in addition to the traditional notion of algorithmic cost. With a given method, we would be able to model both the algorithmic cost of a certain robot behavior as well as the social cost. Let us use the example of the person-robot distance $d_{r p}$. We might observe that people are very receptive, or even negatively affected by the robot being outside of a $5 \mathrm{~cm}$ interval centered on $d_{r p}$ when the robot is close, while a larger error in $d_{r p}$ may go unnoticed when the robot is far. Generally, maintaining better accuracy in navigation requires more computational resources, so the social cost of failing to maintain an accurate position could be formulated as being high when near, and low when far, whereas the algorithmic cost for maintaining an accurate position is the same.

We plan to use the Utility framework of [3] combined with our extensions to model various navigation tasks other than person-following, allowing us to further study HRI during robot navigation, following our iterative design methodology.

\section{REFERENCES}

[1] C. J., "The Quiet Professional: An investigation of U.S. military Explosive Ordnance Disposal personnel interactions with everyday field robots ," Doctoral dissertation University of Washington, 2013. 
[2] A. M. Rosenthal-von der Pütten, F. P. Schulte, S. C. Eimler, S. Sobieraj, L. Hoffmann, S. Maderwald, M. Brand, and N. C. Krämer, "Investigations on empathy towards humans and robots using fMRI," Computers in Human Behavior, vol. 33, pp. 201-212, apr 2014. [Online]. Available: https://linkinghub.elsevier.com/retrieve/pii/S0747563214000090

[3] L. Y. Morales Saiki, S. Satake, R. Huq, D. Glass, T. Kanda, and N. Hagita, "How do people walk side-by-side?: Using a computational model of human behavior for a social robot," Proceedings of the seventh annual ACM/IEEE international conference on Human-Robot Interaction - HRI '12, no. May 2016, p. 301, 2012. [Online]. Available: http://dl.acm.org/citation.cfm?doid=2157689.2157799

[4] J. J. Park and B. Kuipers, "Autonomous person pacing and following with Model Predictive Equilibrium Point Control," Proceedings - IEEE International Conference on Robotics and Automation, no. February 2015, pp. 1060-1067, 2013

[5] M. J. White, "Interpersonal Distance as Affected by Room Size, Status, and Sex," The Journal of Social Psychology, vol. 95, no. 2, pp. 241-249, apr 1975. [Online]. Available: http://www.tandfonline.com/doi/abs/10.1080/00224545.1975.9918710

[6] E. T. Hall, R. L. Birdwhistell, B. Bock, P. Bohannan, A. R. Diebold, M. Durbin, M. S. Edmonson, J. L. Fischer, D. Hymes, S. T. Kimball, W. L. Barre, S. J. Frank Lynch, J. E. McClellan, D. S. Marshall, G. B. Milner, H. B. Sarles, G. L. Trager, and A. P. Vayda, "Proxemics [and comments and replies]," Current Anthropology, vol. 9, no. 2/3, pp. 83-108, 1968. [Online]. Available: http://www.jstor.org/stable/2740724

[7] M. Argyle and J. Dean, "Eye-Contact, Distance and Affiliation," Sociometry, vol. 28, no. 3, p. 289, sep 1965. [Online]. Available: https://www.jstor.org/stable/2786027?origin=crossref

[8] C. Gobl and A. Ni Chasaide, "The role of voice quality in communicating emotion, mood and attitude," Speech Communication, vol. 40, no. 1-2, pp. 189-212, apr 2003. [Online]. Available: https://www.sciencedirect.com/science/article/abs/pii/S016763930200082

[9] A. E. Scheflen and A. Scheflen, Body language and the social order: Communication as behavioral control. Oxford, England: PrenticeHall, 1972

[10] L. Guillaume, V. Aubergé, R. Magnani, F. Aman, C. Cottier, Y. Sasa, C. Wolf, F. Nebout, N. Neverova, N. Bonnefond, A. Nègre, L. Tsvetanova, and M. Girard-Rivier, "Hri in an ecological dynamic experiment: The gee corpus based approach for the emox robot," in 2015 IEEE International Workshop on Advanced Robotics and its Social Impacts (ARSO), June 2015, pp. 1-6.

[11] Y. Sasa, "Intelligence from Socio-Affects of Robot : Dialog Primitives for a Scalable Interaction with a Smart Home Robot," Theses, Université Grenoble Alpes, Jan. 2018. [Online]. Available: https://tel.archives-ouvertes.fr/tel-01945238

[12] L. Tsvetanova, V. Aubergé, and Y. Sasa, "Multimodal breathiness in interaction: from breathy voice quality to global breathy "body behavior quality"," 2017, -. [Online]. Available: https://hal.archivesouvertes.fr/hal-01612322

[13] B. Mutlu and J. Forlizzi, "Robots in organizations," Proceedings of the 3rd international conference on Human robot interaction - HRI '08, no. May 2014, p. 287, 2008. [Online]. Available: http://portal.acm.org/citation.cfm?doid=1349822.1349860

[14] J. Mumm and B. Mutlu, "Human-robot proxemics: Physical and psychological distancing in human-robot interaction," in 2011 6th ACM/IEEE International Conference on Human-Robot Interaction (HRI), March 2011, pp. 331-338.

[15] A. Sciutti, C. Ansuini, C. Becchio, and G. Sandini, "Investigating the ability to read others' intentions using humanoid robots." Frontiers in psychology, vol. 6, p. 1362, 2015. [Online]. Available: http://www.ncbi.nlm.nih.gov/pubmed/26441738 http://www.pubmedcentral.nih.gov/articlerender.fcgi?artid=PMC4563880

[16] D. Vaufreydaz, W. Johal, and C. Combe, "Starting engagement detection towards a companion robot using multimodal features," Robotics and Autonomous Systems, vol. 75, pp. 4-16, jan 2016. [Online]. Available: https://linkinghub.elsevier.com/retrieve/pii/S0921889015000111

[17] T. Munzer, Y. Mollard, and M. Lopes, "Impact of robot initiative on human-robot collaboration," in Proceedings of the Companion of the 2017 ACM/IEEE International Conference on Human-Robot Interaction, ser. HRI '17. New York, NY, USA: ACM, 2017, pp. $217-$ 218. [Online]. Available: http://doi.acm.org/10.1145/3029798.3038373

[18] M. Shiomi, K. Shatani, T. Minato, and H. Ishfguro, "Does a Robot's Subtle Pause in Reaction Time to People's Touch Contribute to Posi- tive Influences?" RO-MAN 2018 - 27th IEEE International Symposium on Robot and Human Interactive Communication, pp. 364-369, 2018.

[19] A. Spalanzani, "Contribution à la navigation autonome en environnement dynamique et humain," Habilitation à diriger des recherches, MSTII, June 2015. [Online]. Available: https://hal.inria.fr/tel-01196699

[20] J. G. Da Silva Filho and T. Fraichard, "Human Robot Motion: A Shared Effort Approach," in European Conference on Mobile Robotics, Paris, France, Sept. 2017. [Online]. Available: https://hal.inria.fr/hal01565873

[21] J. Rios-Martinez, A. Renzaglia, A. Spalanzani, A. Martinelli, and C. Laugier, "Navigating between people: A stochastic optimization approach," Proceedings - IEEE International Conference on Robotics and Automation, no. July 2015, pp. 2880-2885, 2012.

[22] M. Shiomi, F. Zanlungo, K. Hayashi, and T. Kanda, "Towards a Socially Acceptable Collision Avoidance for a Mobile Robot Navigating Among Pedestrians Using a Pedestrian Model," International Journal of Social Robotics, vol. 6, no. 3, pp. 443-455, 2014.

[23] M. Svenstrup, S. T. Hansen, H. J. Andersen, and T. Bak, "Adaptive Human-Aware Robot Navigation in Close Proximity to Humans," International Journal of Advanced Robotic Systems, vol. 8, no. 2, p. 21, jun 2011. [Online]. Available: http://journals.sagepub.com/doi/10.5772/10570

[24] T. Fraichard, R. Paulin, and P. Reignier, "Human-Robot Motion: An Attention-Based Navigation Approach," in IEEE Int. Symp. on Robot and Human Interactive Communication (ROMAN), Edinburgh, United Kingdom, Aug. 2014. [Online]. Available: https://hal.inria.fr/hal01018471

[25] B. Irfan, J. Kennedy, S. Lemaignan, F. Papadopoulos, E. Senft, and T. Belpaeme, "Social Psychology and HumanRobot Interaction: An Uneasy Marriage," Companion of the 2018 ACM/IEEE International Conference on Human-Robot Interaction - HRI '18, pp. 13-20, 2018. [Online]. Available: http://dl.acm.org/citation.cfm?doid=3173386.3173389

[26] Y. F. Chen, M. Everett, M. Liu, and J. P. How, "Socially aware motion planning with deep reinforcement learning," IEEE International Conference on Intelligent Robots and Systems, vol. 2017-Septe, pp. 1343-1350, 2017.

[27] O. A. Ramirez, H. Khambhaita, R. Chatila, M. Chetouani, and R. Alami, "Robots learning how and where to approach people," 25th IEEE International Symposium on Robot and Human Interactive Communication, RO-MAN 2016, vol. 1, pp. 347-353, 2016.

[28] M. Luber, L. Spinello, J. Silva, and K. O. Arras, "Socially-aware robot navigation: A learning approach," IEEE International Conference on Intelligent Robots and Systems, pp. 902-907, 2012.

[29] M. Sebastian, S. B. Banisetty, and D. Feil-Seifer, "Socially-aware navigation planner using models of human-human interaction," in 2017 26th IEEE International Symposium on Robot and Human Interactive Communication (RO-MAN). IEEE, aug 2017, pp. 405-410. [Online] Available: http://ieeexplore.ieee.org/document/8172334/

[30] Z. Wan, X. Hu, H. He, and Y. Guo, "A learning based approach for social force model parameter estimation," in 2017 International Joint Conference on Neural Networks $(I J C N N)$. IEEE, may 2017, pp. 4058-4064. [Online]. Available: http://ieeexplore.ieee.org/document/7966368/

[31] S. S. Honig, T. Oron-Gilad, H. Zaichyk, V. Sarne-Fleischmann, S. Olatunji, and Y. Edan, "Toward Socially Aware Person-Following Robots," IEEE Transactions on Cognitive and Developmental Systems, vol. 10, no. 4, pp. 936-954, 2018.

[32] A. Leigh, J. Pineau, N. Olmedo, and H. Zhang, "Person tracking and following with 2D laser scanners," in 2015 IEEE International Conference on Robotics and Automation (ICRA). IEEE, may 2015, pp. 726-733. [Online]. Available: http://ieeexplore.ieee.org/document/7139259/

[33] H. Zender, P. Jensfelt, and G. M. Kruijff, "Human- and situation-aware people following," in RO-MAN 2007 - The 16th IEEE International Symposium on Robot and Human Interactive Communication, Aug 2007, pp. 1131-1136.

[34] E. Prassler, D. Bank, and B. Kluge, "Motion coordination between a human and a mobile robot," IEEE International Conference on Intelligent Robots and Systems, vol. 2, no. February 2002, pp. 12281233, 2002.

[35] A. Cosgun, D. A. Florencio, and H. I. Christensen, "Autonomous person following for telepresence robots," in 2013 IEEE International Conference on Robotics and Automation. 
IEEE, may 2013, pp. 4335-4342. [Online]. Available: http://ieeexplore.iee.org/document/6631191/

[36] E. Repiso, G. Ferrer, and A. Sanfeliu, "On-line adaptive side-byside human robot companion in dynamic urban environments," IEEE International Conference on Intelligent Robots and Systems, vol. 2017Septe, pp. 872-877, 2017.

[37] R. Murakami, L. Y. Morales Saiki, S. Satake, T. Kanda, and H. Ishiguro, "Destination Unknown: Walking Side-by-Side without Knowing the Goal," Human-Robot Interaction (HRI), Proceedings of the 2014 ACM/IEEE International Conference on, pp. 471-478, 2014. [Online]. Available: http://doi.acm.org/10.1145/2559636.2559665

[38] Y. Morales, T. Kanda, and N. Hagita, "Walking Together: Side by Side Walking Model for an Interacting Robot," Journal of HumanRobot Interaction, vol. 3, no. 2, p. 51, 2014.

[39] D. Karunarathne, Y. Morales, T. Kanda, and H. Ishiguro, "Model of Side-by-Side Walking Without the Robot Knowing the Goal," International Journal of Social Robotics, vol. 10, no. 4, pp. 401-420, sep 2018. [Online]. Available: http://link.springer.com/10.1007/s12369-017-0443-6 